\title{
Riesenzellarteriitis der Arteria temporalis links - Ausgedehnte Skalpnekrose bei einer 71-jährigen Patientin
}

\section{Giant-Cell Arteriitis-Horton (Magath-Brown Disease) in an 71-Year Old Female}

Autoren

Institut
K. Braunert, S. Weimann, C. S. L. Müller, J. Reichrath, T. Vogt

Klinik für Dermatologie, Venerologie und Allergologie, Universitätsklinikum des Saarlandes, Homburg/Saar

\section{Bibliografie}

DOI http://dx.doi.org/

10.1055/s-0030-1256124

Akt Dermatol 2011; 37:

24-26 @ Georg Thieme

Verlag KG Stuttgart · New York

ISSN 0340-2541

Korrespondenzadresse

Karin Braunert

Universitätsklinikum

des Saarlandes

Klinik für Dermatologie,

Venerologie und Allergologie

Kirrberger Str. 1

66421 Homburg/Saar

karin.braunert@uks.eu

\section{Zusammenfassung \\ $\nabla$}

Die Arteriitis temporalis ist eine relativ seltene segmentale, granulomatöse, obliterierende Vasculitis. Pathogenetisch findet sich eine granulomatöse Riesenzellarteriitis im Bereich von Media und

\section{Kasuistik}

Die 71-jährige Patientin stellte sich vor aufgrund des Verdachtes auf einen therapieresistenten, nekrotisierenden Herpes zoster der Kopfhaut. Die hautfachärztliche Konsultation erfolgte wegen persistierender Schmerzen sowie einer Progredienz der Hautveränderungen trotz 11-tägiger stationärer Behandlung in einem heimatnahen Krankenhaus. Zusätzlich berichtet die Patientin über seit 2 Monaten aufgetretene Sehstörungen. So habe sie beim Autofahren die Fahrbahnmarkierung teilweise doppelt gesehen. Eine augenärztliche Untersuchung, ein CCT sowie ein EEG hätten keinen richtungsweisenden Befund ergeben. Seit mehreren Wochen bestünden auch starke Kopfschmerzen („tausend Nadelstiche im Kopf“).

Vor ca. einem Monat sei an der Stirn links zunächst ein Hämatom, dann eine Krustenbildung mit Nässen aufgefallen. Es erfolgte eine heimatnahe 11-tägige stationäre antivirale Therapie und Analgesie unter der Verdachtsdiagnose eines nekrotisierenden Herpes zoster. Nach der Entlassung hätten sich der Hautbefund und die Schmerzen zusätzlich weiter verschlechtert.

Die dermatologische Eigen- und Familienanamnese war unauffällig. An Vorerkrankungen waren eine absolute Arrhythmie (mit Marcumar-Therapie), eine arterielle Hypertonie, ein Z.n. intraorbitaler Gefäßverschluss rechts 2005 und Myokardinfarkt 2002 bekannt.

Bei Aufnahme sah man im Bereich der gesamten Kopfhaut unregelmäßig-bizarr konfigurierte, großflächige, teils blutig-krustös belegte tiefe
Adventitia der kleinen und mittleren Arterien. Die Diagnose ist klinisch oft nicht leicht zu stellen. Wir stellen einen Fall einer Riesenzellarteriitis der Arteria temporalis links bei einer 71-jährigen Patientin vor, welche zuvor unter der Verdachtsdiagnose eines Herpes zoster behandelt wurde.

Ulzerationen mit Überschreitung der Mittellinie von links nach rechts ( $\bullet$ Abb. 1).

Temporal ließen sich pulslose, strangartige Resistenzen tasten. Das gesamte übrige Integument und die Schleimhäute waren unauffällig.

\section{Dermatohistologie}

$\nabla$

Nach Doppler-sonograpfischer Sicherung pulsloser strangförmiger Gefäßstrukturen temporal erfolgte eine Exzisionsbiopsie aus der A. temporalis links. Der histologische Befund zeigte eine komplette Thrombosierung des arteriellen Gefäßlumens mit transmuraler Durchsetzung der Gefäßwand durch ein lymphoplasmazelluläres Entzündungsinfiltrat mit deutlicher histiozytärer Beteiligung und zahlreichen mehrkernigen Riesenzellen mit Phagozytose von elastischen Fasern („Elastophagozytose“) ( $\bullet$ Abb. 2).

\section{Weitere Diagnostik, Therapie und Verlauf $\nabla$}

Wir stellten die Patientin konsiliarisch in der Augenklinik vor. Es zeigten sich an der peripheren Netzhaut am linken Auge kleine Blutungen ohne Handlungsbedarf. Die Patientin wurde aufgrund der Doppelbilder zudem in der Klinik für Neurologie vorstellig. In der Kernspintomografie zeigten sich die bekannten Weichteildefekte, zudem bestand der Verdacht auf eine knöcherne Beteiligung links, diese wurde jedoch durch ein CCT ausgeschlossen. In der Duplexsonografie stellte sich 

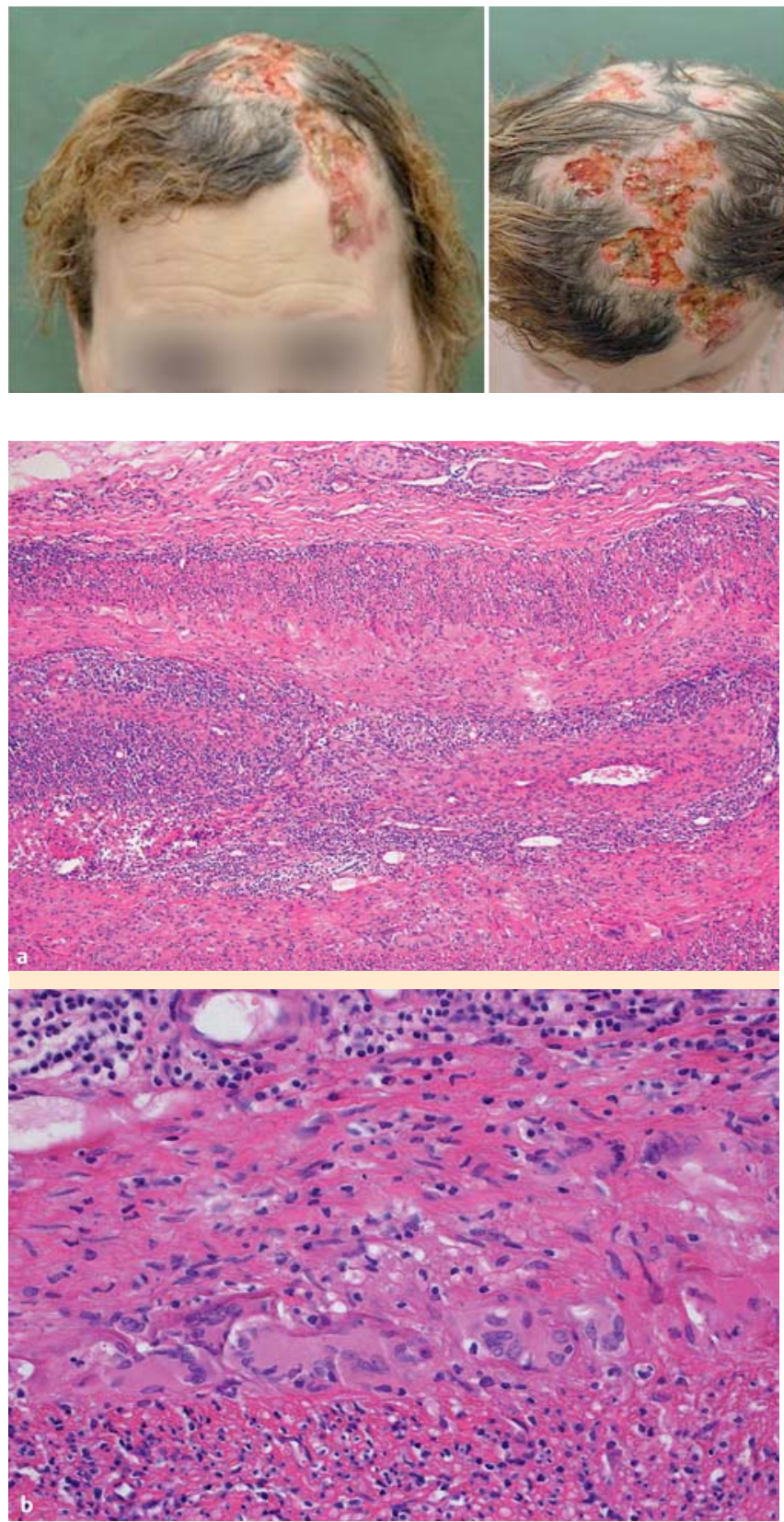

Abb. 2 Längsschnitt durch die Arteria temporalis links mit vollständiger Okklusion des Gefäßlumens sowie mehrkernigen Riesenzellen in der Gefäßwand (Hämatoxylin/Eosin $100 \times$ und $400 \times$ ).

die Arteria temporalis links im temporalen bis frontalen Verlauf vollständig ohne Fluss dar.

Wir initiierten eine interne Therapie mit Methylprednisolon (Urbason ${ }^{\circledR}$ ) $80 \mathrm{mg} / \mathrm{d}$ i.v. und eine interne Antibiose mit Ciprofloxacin bei bakterieller Superinfektion der kutanen Ulzerationen. Zur Schmerzmedikation wurden Targin ${ }^{\circledR}$ (Oxycodon, Naloxon), Sevredol ${ }^{\circledR}$ (Morphinsulfat) und Novalgin ${ }^{\circledR}$-Tropfen (Metamizol) verabreicht. Hierunter kam es zu einer zügigen Abheilungstendenz sowie einer guten Kontrolle der Schmerzsymptomatik. Das Kortikosteroid konnte langsam auf $10 \mathrm{mg} / \mathrm{d}$ im täglichen Wechsel mit $5 \mathrm{mg} / \mathrm{d}$ reduziert werden (im Verlauf von 16 Wochen).

Lokal behandelten wir initial mit Lavasept-Umschlägen sowie mit Diprogenta ${ }^{\circledR}$-Creme (Betamethasondiproprionat und Gentamicinsulfat) in der Umgebung. Im Verlauf Wunddébridement und Applikation von Fucicort ${ }^{\circledR}$-Creme (Fusidinsäure). Hierunter

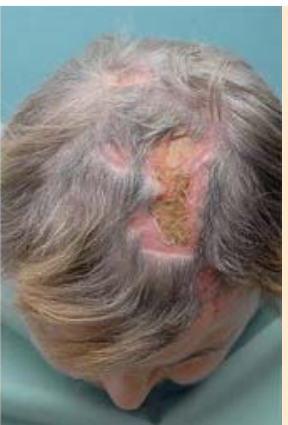

Abb. 1 Links und Mitte: unregelmäßig konfigurierte, großflächige, teils blutig-krustös belegte tiefe Ulzerationen mit Überschreitung der Mittellinie. Rechts: Verlaufskontrolle nach 16 Wochen.

zeigte sich eine sehr gute Befundbesserung mit Zunahme der Wundgranulation ( $\bullet$ Abb. 1).

\section{Diskussion}

\section{$\nabla$}

Bei der vorgestellten Patientin liegt eine Riesenzellarteriitis der Arteria temporalis links vor. Synonym werden die Bezeichnungen Arteriitis temporalis, Arteriitis cranialis, Morbus Horton, HortonSyndrom oder Horton-Magath-Brown-Syndrom verwendet. Die Erstbeschreibung der Erkrankung erfolgte in den Jahren 1890 (Hutchinson) bzw. 1934 (Horton).

Es handelt sich um eine relativ seltene segmentale, granulomatöse, obliterierende Vaskulitis. Die jährliche Inzidenz beträgt ca. 90/1000000 Einwohner, bei > 50-Jährigen jedoch bis 170/ 1000000 Einwohner [1]. Ätiologisch werden ein T-Zell-abhängiges (Auto-)Immungeschehen bei genetischer Prädisposition und Störungen im Endothelin-System [2], möglicherweise durch Infekte ausgelöst, diskutiert. Pathogenetisch findet sich eine granulomatöse Riesenzellarteriitis im Bereich von Media und Adventitia der befallenen Arterienabschnitte. Klinisch imponieren Hautrötungen und Schwellungen über geschlängelter, druckschmerzhaft verhärteter, pulsloser Temporalarterie [4]. Später können Kopfhautnekrosen hinzutreten. Die Diagnose ist klinisch oft nicht leicht zu stellen. Klinische Differenzialdiagnosen umfassen v.a. den Herpes zoster bzw. Zosterneuralgie, Arteriosklerose, Thrombangitis obliterans und Ergotismus (durch Migränetherapeutika) [1].

Die Diagnose ist durch folgende ACR-Kriterien (American College of Rheumatology) (Hunder et al. 1990) zu erhärten (bei 3 von 5 zutreffenden Kriterien: Sensitivität 75-90\%, Spezifität 90-93\%):

- Alter $>50$ Jahre

- (Schläfen-)Kopfschmerzen, evtl. Kauschmerzen („Masseterschmerz“)

- Auffällige A. temporalis (Druckschmerz u./o.

Pulsabschwächung/Pulslosigkeit)

- $\mathrm{BSG}>50 \mathrm{~mm}$ in der ersten Stunde

- Histologisch Veränderungen der A. temporalis: segmentale

Vasculitis („skip lesions“) [3]

Allgemeinsymptome wie Fieber, neu aufgetretene ein- oder doppelseitige Kopfschmerzen (v. a. temporal und im Stirnbereich (ca. $50 \%$ ), Arthralgien, Myalgien (Polymyalgia rheumatica bei 50\% der Patienten) und Gewichtsverlust [3] sind beschrieben. Ein Erythema nodosum oder eine Urtikaria kommen in etwa $15 \%$ der Fälle vor sowie Rötungen im Bereich der strangartig verdickten A. temporalis. Bei Zungenbeteiligung (Arteriitis lingualis) werden Nekrosen, Blasen und Erytheme angegeben [1].

Extrakutane und diverse atypische Manifestationen kommen vor, z.B. mit Armclaudicatio (50\%), Augenbeteiligung (30\%), orthostatisch bedingtem Schwindel (40\%), Krampfanfällen und 
Hemiparesen ( $<10 \%$ der Fälle) oder in etwa $10 \%$ der Fälle eine Claudicatio der unteren Extremitäten sowie Aorteninsuffizienzen und Aortenaneurysmen $[1,4]$.

In etwa $30 \%$ der Fälle treten Sehstörungen auf, ein Hauptrisiko der Arteriitis temporalis ist (bei Mitbeteiligung der Arteria ophthalmica) die Erblindungsgefahr (unbehandelt ca. 30\%). Warnzeichen sind die oft zitierte Amaurosis fugax oder Augenschmerzen. Therapieziel ist die Reduzierung der Gefäßwandentzündung. Therapeutisch ist ein gutes Ansprechen häufig nur auf Glukokortikosteroide mit Erhaltungsdosis über 1-2 Jahre zu sehen. Es kommt in der Regel zu kompletten Remissionen nach 6-24 Monaten, Rezidive und chronische Verläufe sind selten. Bereits bei einseitiger Augensymptomatik ist die Steroiddosis zu erhöhen auf 1,5-2 mg/kg KG/Tag Prednisolonäquivalent (im Vergleich zu 1,0-1,5 mg ohne Augenbeteiligung). Bei Rezidiven unter der Behandlung ist u.U. die zusätzliche Gabe von Cyclophosphamid ( $2 \mathrm{mg} / \mathrm{kg} \mathrm{KG} / \mathrm{Tag}$ ) notwendig, bei etwa $4 \%$ der Patienten tritt daraufhin eine Besserung ein. Statt Cyclophosphamid kann eine additive Therapie mit Methotrexat erwogen werden [1]. Als kortikosteroidsparende Alternative wird künftig eine Hemmung des Endothelin-(ET-)Rezeptors erwartet. Eine Studie mit 10 an GCA erkrankten Patienten zeigte eine Korrelation zwischen der Aktivität des ET-Rezeptors und dem Grad der systemischen Entzündung [2].

Zum Monitoring der Krankheitsaktivität bieten sich Kontrollen von BSG und CRP an [3], wobei das C-reaktive Protein als Verlaufsparameter aussagekräftiger ist.

\section{Danksagung}

Besonderer Dank gilt der ltd. MTA, Frau Anne Kerber, für die vorzüglichen Schnittpräparate.

\section{Abstract}

\section{Giant-Cell Arteriitis-Horton (Magath-Brown Disease) in an 71-Year Old Female \\ $\nabla$}

The Giant-cell arteritis-Horton (GCA, -Magath-Brown disease) is a rather rare segmental, granulomatous obliterating vasculitis. Pathogentically a granulomatous inflammation of the media and adventitia of small to medium sized arteries is found. The diagnosis might be missed and can be difficult. Here we present the case of a 71-year old patient, who was presenting to our outpatient clinic as „herpes zoster“.

\section{Literatur}

1 Cantini F, Niccoli L, Nannini C et al. Diagnosis and treatment of giant cell arteritis. Drugs Aging 2008; 25: 281-297

2 Dimitrijevic I, Andersson C, Rissler P, Edvinsson L. Increased tissue endothelin-1 and endothelin-B receptor expression in temporal arteries from patients with giant cell arteritis. Ophtalmology 2010; 117 (3): $628-636$

3 Ness T, Auw-Hädrich C, Schmidt D. Temporal arteritis (giant cell arteritis). Clinical picture, histology and treatment. Ophtalmologe 2006; 103 (4): $296-301$

4 Serrano-Falcón C, Del Mar Serrano-Falcón M, Callejas-Rubio JL, SerranoOrtega S. Scalp necrosis as a manifestation of temporal arteritis. International Journal of Dermatology 2010; 49: 466-467 